\title{
Relato verbal de idosos para geração de informações sobre as categorias usadas para auto-avaliação dos efeitos do tratamento hidroterapêutico*
}

\section{Verbal report of seniors for generation of information on the categories used for self-evaluation of the effects of hydrotherapeutic treatment}

\author{
Juliana Monteiro Candeloro ${ }^{1}$, Fátima Aparecida Caromano²
}

\begin{abstract}
CANDELORO, J. M.; CAROMANO, F. A. Relato verbal de idosos para geração de informações sobre as categorias usadas para auto-avaliação dos efeitos do tratamento hidroterapêutico. Rev. Ter. Ocup. Univ. São Paulo, v. 16, n. 3, p. 131-136, set./dez., 2005.

RESUMO: Este trabalho vem demonstrar como o relato verbal pode ser uma rica fonte de dados sobre as categorias de auto-avaliação da evolução clínico-funcional estabelecidas pelos sujeitos em relação à intervenção hidroterapêutica efetuada. Após a análise dos relatos de 16 mulheres de um programa de hidroterapia encontrou-se sete categorias de referências de evolução clínico-funcional: melhora no desempenho ao realizar atividades de hidroterapia; melhora de sensações e percepções corporais; melhora funcional; melhora no quadro álgico; melhora na execução das atividades diárias e diminuição do uso de medicação. Esses relatos podem ser utilizados como feedback para o fisioterapeuta ao avaliar o critério de referência do paciente, auxiliando na criação de uma linguagem comum e possibilitando o uso de reforçadores mais específicos.
\end{abstract}

DESCRITORES: Educação especial. Hidroterapia. Envelhecimento. Mulheres. Auto-avaliação (Psicologia). Terapia por exercício.

\footnotetext{
* Este artigo faz parte da tese de mestrado de Juliana Monteiro Candeloro.

${ }^{1}$ Mestranda da Ciências e Reabilitação da Faculdade de Medicina da USP, especialista em Hidroterapia pela Universidade Bandeirante de São Paulo.

${ }^{2}$ Prof. Dra. do Curso de Fisioterapia da Faculdade de Medicina da USP.

Endereço de correspondência: LaFi-ReaCom - Laboratório de Fisioterapia e Reatividade Comportamental. Rua Cipotânea, 51; CEP 05360-000. Cidade Universitária da USP- São Paulo, Brasil. juliana@ poolterapia.com.br
} 


\section{INTRODUÇÃO}

$\mathrm{O}$ relato verbal pode ser usado como fonte de dados. Atualmente, é amplamente utilizado por psicólogos, constituindo a base de entrevistas clínicas e experimentais, levantamentos e avaliações padronizadas (KERBAUY, 2000). Na avaliação fisioterapêutica, principalmente na anamnese, muitos dados são coletados por meio do relato verbal dos pacientes. Estes relatos têm sido essenciais na obtenção de informações, e podem ser uma via de acesso dos pesquisadores aos comportamentos e estados mentais que não podem ser mensurados (DAMASIO, 2004).

O relato verbal ocorre em situações de interlocução, indicando pelo menos em parte, processos subjetivos de quem relata. Podem ocorrer modificações nos relatos devido à interação de quem está relatando com o interlocutor. $\mathrm{O}$ paciente esteve em contato com um estado de coisas que $o$ ouvinte, ou pesquisador, não teve acesso. Existe a possibilidade de distorções deliberadas dos fatos por parte do relator. Este, também, pode não ter conhecimento da relevância dos fatos envolvidos no seu comportamento. Mesmo considerando estas possibilidades, a situação de entrevista ou aplicação de questionário pode fornecer pistas importantes para atingir os objetivos do pesquisador e também, pode ajudar a pessoa a lembrar detalhes, tornando o relato mais acurado (MARQUES; SIMÃO, 1995).

Neste estudo, aplicou-se um questionário com uma única questão aberta, para pesquisar e categorizar as referências de evolução clínico-funcional estabelecidas por idosos que participaram de um programa de 16 semanas de hidroterapia, elaborado para reeducação funcional, visando uma população sedentária, saudável e na faixa etária de 65 a 70 anos.

A aplicação e avaliação deste programa de hidroterapia justificam-se pela necessidade apresentada por idosos de manter e ou recuperar força (SKELTON et al., 1995; BEISSNER et al, 2000), mobilidade (WALKER, 1984; THOMPSON, 1994; DALEY et al., 2000) e equilíbrio, comumente diminuídos em função do processo de envelhecimento. Este tipo de intervenção está associada à atividade prazerosa de relaxamento, sem ocorrência de sobrecarga articular (WHITE, 1999; KOURY, 2000).

Por meio de avaliação funcional (mensuração de força utilizando dinamômetro e medida de flexibilidade por de testes fotográficos de mobilidade) foi possível verificar que o programa proposto produziu melhoras estatisticamente significativas na força e na flexibilidade.

A avaliação funcional gerou dados numéricos, informando o fisioterapeuta sobre o efeito do tratamento clínico e, não gerou dados referentes à funcionalidade ou aspectos emocionais e sociais.

Com base no exposto, este estudo teve como objetivo analisar e categorizar relatos verbais gerando uma fonte de dados para descrever a percepção dos idosos em relação a evolução clínico-funcional após se submeterem a um programa hidroterapia.

\section{MÉTODO}

Este estudo teve uma abordagem qualitativa, que segundo Minayo (1994) é um tipo de pesquisa que trabalha com o universo de significados, motivos, aspirações, valores e comportamentos, isto é, aborda fenômenos que não podem ser reduzidos unicamente a informações quantitativas. Segundo Turato (2003), a pesquisa qualitativa envolve o entender e o interpretar significados que o sujeito da pesquisa dá a determinado fenômeno ou comportamento. Neste contexto, o que a pesquisa expressa é a visão do entrevistado e o pesquisador é reconhecido como principal instrumento de investigação (ALVES, 1991; THIOLLENT, 1984) daí a necessidade de conhecimento profundo do assunto abordado e treinamento na técnica de realização de observação direta e/ou aplicação de entrevista.

Nesta pesquisa, optou-se pela entrevista semiestruturada, que permitiu a elaboração de um sistema de categorias. Segundo Deslandes (2002) este método permite a reconstrução de uma realidade social, por categorias, que se identificam por possuir características específicas.

A expressão de porcentagem entre sujeitos e respostas fornece uma visão básica quantitativa, que tem por objetivo único complementar as informações fornecidas pelo estudo qualitativo. Neste sentido, a pesquisa busca exprimir as relações de dependência entre variáveis, identificando os objetos do estudo e permitindo realizar deduções e correlações (PORTELA, 2004).

\section{Sujeitos}

Participaram do estudo dezesseis mulheres com idade entre 65 e 70 anos, voluntárias, sedentárias, que receberam orientação e autorização médica para desenvolver atividade física e que concordaram com o termo de consentimento pós-informado.

\section{Local}

A coleta de dados foi realizada na Escola de Natação Pool Center, localizada em São Paulo, no bairro São Judas e o desenvolvimento do projeto foi desenvolvido no LaFiReaCom - do Curso de Fisioterapia da USP. 


\section{Material}

Piscina de $4 \mathrm{~m} \times 2 \mathrm{~m}$, com profundidade de $1,3 \mathrm{~m}$, gravador de fita $\mathrm{K} 7$ e questionário com questão única aberta, desenvolvido para este estudo.

\section{Procedimentos}

Este estudo foi realizado inicialmente com a população de 22 idosos, sendo que 16 deles concluíram o programa de hidroterapia com duração de 16 semanas (32 sessões), elaborado para ganho de força e flexibilidade, com sessões realizadas duas vezes por semana, em duplas, com uma hora de duração. Foram realizados testes de força e flexibilidade antes do programa hidroterapêutico (pré-teste) e no final das 32 sessões (pós-teste), com objetivo de avaliação fisioterapêutica do tratamento. Os participantes foram orientados a não praticar outro tipo de atividade física durante a participação no programa. A avaliação fisioterapêutica mostrou diferença estatisticamente signi- ficativa de força e flexibilidade - dados desconhecidos do pesquisador e dos sujeitos no momento da aplicação do questionário. Desta forma as respostas do questionário não puderam ser influenciadas por estas informações.

Aplicou-se um questionário com pergunta única aberta após a última sessão do tratamento (32 sessão): “O que você acha que melhorou na sua rotina desde o início do tratamento com a hidroterapia?".

Os relatos foram gravados, transcritos, analisados e, posteriormente elaborou-se um conjunto de categorias de evolução clínico-funcional.

\section{RESULTADOSE DISCUSSÃO}

A partir do estudo das respostas do questionário, foram elaboradas categorias relacionadas aos fatores de evolução clínico-funcional referidos pelos idosos. Também avaliou-se o número de relatos por categoria. Os resultados encontrados estão descritos na Tabela 1.

Tabela 1 - Categorias, quantidade de relato/categoria e, número máximo e mínimo de relato por relato/sujeito.

\begin{tabular}{l|c|c|c}
\hline Relatos & $\begin{array}{l}\mathrm{N}^{\circ} \text { de participantes que } \\
\text { referiram estas categorias }\end{array}$ & $\begin{array}{l}\text { Quantidade de relatos referentes } \\
\text { a esta categoria }\end{array}$ & $\begin{array}{l}\mathrm{N}^{\circ} \text { máximo e mínimo de } \\
\text { relato desta relato/sujeito }\end{array}$ \\
\hline $\begin{array}{c}\text { 1. Informam sobre o próprio desempenho } \\
\text { ao realizar atividades da hidroterapia }\end{array}$ & 5 & 5 & $1 ; 1$ \\
$\begin{array}{l}\text { 2. Informam sobre sensações e percepções } \\
\text { corporais }\end{array}$ & 16 & 28 & $4 ; 3$ \\
3. Informam sobre melhoras funcionais & 13 & 26 & $3 ; 1$ \\
4. Informam melhora do quadro álgico & 12 & 20 & $3 ; 1$ \\
5. Informam sobre melhora na realização de & & 15 & $2 ; 1$ \\
$\quad$ atividades diárias & 10 & 11 & $2 ; 1$ \\
6. Informam melhoras emocionais & 8 & 5 & $1 ; 1$ \\
7. Diminuição de uso de medicação & 5 & & \\
\hline
\end{tabular}

Os 16 participantes, independentemente da forma com que avaliou sua evolução, referiram algum tipo de melhora. Das sete categorias encontradas as mais citadas foram a 2, 3 e 4, as menos citadas foram as categorias 1 e 7 e as categorias de importância intermediária para os idosos foram as 5 e 6 (Tabela 1 ).

Todos os participantes utilizaram a categoria que informa sobre as sensações e percepções do próprio corpo, tendo sido encontrado 28 citações com máximo de relato/ sujeito quatro e mínima de três. Observou-se que esta categoria pode ser percebida como resultado direto da hidroterapia, onde, através do aprendizado de exercícios eles puderam reconhecer o funcionamento do próprio corpo. Como exemplo desta categoria, temos:

"Sinto o corpo mais leve, os nervos destravados, estou com maior disposição e mais animada."

"Minha silhueta diminuiu."

A segunda categoria mais citada foi a que informa sobre a melhora funcional do paciente, 13 sujeitos relataram esta categoria, totalizando 26 citações, onde o numero de relato/ sujeito máximo foi de três e mínimo de um. Na fisioterapia a melhora funcional é um dos referenciais mais importantes na evolução do tratamento. Como exemplo destas citações tem-se: 


\author{
"Controlo melhor a bexiga e o intestino está \\ funcionando melhor.” \\ "Durmo melhor, sem interrupção. A freqüência de \\ insônia diminuiu." \\ íram" \\ "O tornozelo não bambeia mais e as cãibras diminu-
}

"Não puxo mais o pé com a mão para colocar a meia."

A terceira categoria mais citada, informa sobre a melhora do quadro álgico, onde 12 sujeitos referem esta categoria com 20 citações. O número máximo de relato/sujeito foi três e mínimo de uma. Outro objetivo de grande importância na fisioterapia é a diminuição do quadro álgico dos pacientes e segundo Heuvelen ${ }^{6}$ estas dores aparecem pelo desuso e não por alguma enfermidade propriamente dita. Segue exemplos desta categoria:

"Não sinto mais dores no joelho que foi operado há três anos atrás."

Nas categorias com citações intermediárias, encontrou-se uma categoria que informa sobre a melhora na realização de atividades diárias, sendo que 10 idosos fizeram este tipo de relato, com total de 15 relatos. O número máximo de relato/sujeito foi dois e mínimo de um. O desempenho físico em tarefas diárias é um forte fator relacionado com a mortalidade, longos períodos de internações ou dependência (HUNTER, 1995). O reconhecimento de melhora nesta categoria é indicador de melhora na independência física (TINETTI, 1986; ERICSSEN et al., 1998).

Da mesma maneira que o fisioterapeuta se preocupa com a qualidade do desempenho nas atividades diárias, freqüentemente os idosos se utilizam destas para estabelecer objetivos a serem atingidos com o tratamento fisioterapêutico. Como exemplo desta categoria tem-se:

"Não canso tanto quando subo ladeiras e subo com maior velocidade, sem pequenas paradas." casa."

"Estou mais disposta para fazer as atividades da

"Não tropeço mais na rua."

"Agora quando vou rezar levanto os dois braços, antes não levantava o braço esquerdo."

A segunda categoria com citação de freqüência intermediária refere-se a melhoras emocionais. Oito idosos relataram esta categoria e 11 relatos foram feitos. O numero máximo de relato/idoso foi dois e mínimo de um. Segundo Grove e Gordon (1992), a participação em programas de hidroterapia pode aliviar sentimentos de depressão e isolamento e ajuda a preservar a auto-estima. A melhora no quadro emocional por meio da hidroterapia é relevante, já que muitos idosos apresentam quadro de depressão e tristeza. Como exemplo destes relatos temos:

"É bom para o estresse e descansa a mente e choro menos agora."

"Estou mais calma e tranqüila."

"Estou melhor da cabeça."

Dentro das categorias menos citadas encontra-se a categoria que informa a melhora do desempenho ao realizar as atividades durante a hidroterapia. Cinco sujeitos com um relato cada, com numero máximo e mínimo de relato/idoso de um, fizeram este tipo de relato. Como exemplo temos:

"Não dói tanto a perna quando faço este alongamento (músculo quadríceps).” dor.”

"Não conseguia rodar o ombro, agora consigo sem

A outra categoria menos citada foi a diminuição no uso da medicação. Cinco sujeitos, com um relato cada e numero máximo e mínimo de relato/idoso de um fizeram esta citação. Esta mudança referida reflete na economia destes idosos, pois muitos deles nesta faixa etária não possuem um trabalho rentativo e dependem muitas vezes da ajuda dos familiares. A diminuição de gastos com medicação pode resultar em pequenos investimentos que podem melhorar sua qualidade de vida, bem como dispensar serviços de ajudantes e enfermagem. Como exemplo de relatos desta categoria, temos:

"Não tomei a medicação do joelho que o médico indicou, não doeu mais."

"Não tomo mais o remédio da coluna."

"Não tomo mais remédio de artrose."

A observação destas melhoras, avaliação funcional e relatos verbais mostradas neste estudo, vem ratificar o fato do processo de envelhecimento associado ao sedentarismo poder ocasionar independências e internações (devido à doenças como pneumonia e fraturas por quedas). O exercício físico pode reverter parcialmente ou totalmente esses efeitos repercutindo na independência, funcionabilidade, na quantidade de dor e na diminuição de internações clínicas e na ingestão de medicamentos (HEUVELEN et al., 2000).

\section{CONCLUSÃO}

Neste estudo foram encontradas sete categorias de relatos verbais de auto-avaliação dos efeitos do tratamento de hidroterapia. As mais relatadas foram os que informaram sobre as sensações e percepções corporais, sobre as melhoras funcionais e diminuição do quadro álgico. As menos citadas foram sobre a melhora ao desempenhar as atividades de hidroterapia e diminuição do uso medicação. Normalmente, estas categorias que foram mais citadas são 
acompanhadas de perto pelo fisioterapeuta. As categorias menos citadas confirmam os benefícios da hidroterapia não só no sistema musculoesquelético como também na qualidade de vida desta população.

Conclui-se que atividades desenvolvidas em programas de hidroterapia traz benefícios físicos, mensurados pelo fisioterapeuta e benefícios funcionais, emocionais, sociais e econômicos, observados e relatados pelo paciente. A partir do conhecimento destas categorias é possível para o fisioterapeuta incentivar diferentes observações por parte dos sujeitos, usar os relatos verbais como feedback (linguagem mais adequada às categorias utilizados pelos pacientes) e para redirecionar e dar continuidade ao trabalho.

CANDELORO, J. M.; CAROMANO, F. A. Verbal report of seniors for generation of information on the categories used for self-evaluation of the effects of hydrotherapeutic treatment. Rev. Ter. Ocup. Univ. São Paulo, v. 16, n. 3, p. 131-136, set./dez., 2005.

\begin{abstract}
This study comes to demonstrate, as the verbal report can be a rich source of data on the categories of self-evaluation of the clinical-functional evolution established by the subjects in relation to the intervention with hydrotherapy. After the analysis of the 16 woman of a hydrotherapy program reports we met functional clinical seven categories of evolution references: improves in the movements when accomplishing hydrotherapy activities, improves of the sensations and corporal perceptions, functional improves, improves of the level of the pain, improves in the execution of the daily activities and decrease of the medication in use. Those reports can be used as feedback for the physiotherapist when evaluating the approach of the patient's reference, aiding in the creation of a common language and facilitating the use of more specific reinforcement.
\end{abstract}

KEY WORDS: Education, special. Hydrotherapy. Anging. Women. Self assessment (Psychology). Exercise therapy.

\title{
REFERENCIAS
}

ALVES, J. A. 1991. O planejamento de pesquisas qualitativas em educação. Cad. Pesq., v. 77, p. 53-61.

BEISSNER, K. L.; COLLINS, J. E.; HOLMES, H. Muscle force and range of motion as predictors of function in older adults. Phys. Ther., v. 80, n. 6, p. 556-563, 2000.

DALEY, M. J.; SPINKS, W. L. Exercise, mobility and aging. Sports Med., v. 29, n. 1, p. 1-12, 2000.

DAMASIO, A. R. Como o cérebro cria a mente. Scientific Am. - Brasil, v. 4, n. 6, p. 11.

DESLANDES, S. F. A construção do projeto de pesquisa. In: MINAYO, C. S. (Org.). Pesquisa social: teoria, método e criatividade: método e criatividade. Rio de Janeiro: Vozes, 2002. Cap.2

ERIKSSEN, G.; LIESTOL, K.; BJORNHOLT, J.; THAULOW, E.; SANDVIK, L; ERIKSSEN, J. Changes in physical fitness and changes in mortality. Lancet, v. 352, p. 759-762, 1998.

GROVE, J. R.; GORDON, A. M. D. The psychological aspects of injury in sport. In: BLOOMFIELD, J.; FRICKER, P. A.; K. D. FITCH, K. D. (Eds.). Textbook of science and medicine in sport. Australia: Blackwell Scientific Publications, 1992.

van HEUVELEN, M. J.; KEMPEN, G. I.; BROUWER, W. H.; de GREEF, M. H. Physical fitness related to disability in older persons. Gerontology, v. 46, n. 6, p. 333-341, 2000.

HUNTER, G. R.; TREUTH, M. S.; WEINSIER, R. L.; KEKESSZABO, T.; KELL, S. H.; ROTH, D. L.; NICHOLSON, C. The effects of strength conditioning on older women ability to perform daily tasks. Am. Geriatr. Soc., v. 43, n. 7, p. 756-760, 1995.

KERBAUY, R. R, et al. Aspectos teóricos, metodológicos e de formação em análise do comportamento e terapia cognitivista. In: Sobre comportamento e cognição. Santo André: ARBytes, 2000. v. 1 , p. $148-163$.

KOURY, J. M. Programa de fisioterapia aquática - um guia para a reabilitação ortopédica. São Paulo: Manole, 2000.

MARQUES, A. P.; SIMÃO, L. M. Análise de relatos verbais de 
um grupo de religiosas sobre sua participação em sessões fisioterápicas. Rev. Fisioter. Univ. São Paulo, v. 2, n. 1, p. 13$21,1995$.

MINAYO, M. C. S. Pesquisa social: teoria, método e criatividade. Rio de Janeiro: Vozes, 1994.

PORTELA, G. L. Abordagens teórico-metodológicas. Projeto de pesquisa no ensino de letras para o Curso de Formação de Professores da UEFS, 2004. Disponível em: http://www.uefs.br/ disciplinas/let318/abordagens_metodológicas.rtf. Acesso em: 28 dez. 2005.

SKELTON, D. A.; YONG, A.; GREIG, C. A.; MALBUT, K. E. Effects of resistance training on strength, power, and selected functional abilities of women aged 75 and older. Am. Geriatr. Soc., v. 43, p. 1081-1087, 1995.

TINETTI, M. E. Performance-oriented assessment of mobility problems in elderly patients. Am. Geriatr. Soc., v. 34, p. 119-

\section{$125,1986$.}

THIOLLENT, M. J. M. Aspectos qualitativos da metodologia de pesquisa com objetivos de descrição. Cad. Pesq., v. 49, p. 45-50, 1984.

THOMPSON, L. V. Effects of age and training on skeletal muscle physiology and performance. Phys. Ther., v. 74, n. 1, p. 71-75, 1994.

TURATO, E. R. Tratado de metodologia da pesquisa clínicoqualitativa. Petrópolis: Vozes, 2003.

WALKER, J. M.; SUE, B.; MILES-ELKOUSY, N.; FORD, G.; TREVELYAN, H. Active mobility of the extremities in older subjects. Phys. Ther., v. 64, n. 6, p. 919-923, 1984.

WHITE, T.; SMITH, B. S. The efficacy of aquatic exercise in increasing strength. Sports Med. Training Rehabil., v. 9, n. 1, p. 51-99, 1999. 